\title{
Keep nanotechnology in mind
}

\author{
Advances in nanotechnology and materials science suggest that a paradigm shift in computation may be \\ closer than we think.
}

\begin{abstract}
Machine learning - the implementation of computational tasks that involve learning from experience - is inevitably inspired by the working principles of biological brains. However, the von Neumann architecture characteristic of conventional computers is intrinsically different from the brain's architecture. Aside from macroscopic differences such as the overall digital nature of the signal processing, opposed to the brain's overall analog approach, the former architecture suffers from an inefficient physical separation between the memory and central processor units.

To clarify the importance of machinelearning algorithms, researchers in the fields of high-energy physics and astronomy already exploit them routinely to extract extremely weak signals buried in noise - the recent detection of gravitational waves by the LIGO Scientific Collaboration being just the latest, groundbreaking example
\end{abstract}

(R. Biswas et al., Phys. Rev. D 88, 062003; 2013). Now, a drastic paradigm shift is needed for the whole architecture of the underlying hardware if machine learning is to become even more efficient and effective. This motivates the recent efforts towards so-called neuromorphic computation, that is, the implementation of algorithms by means of hardware architectures mimicking the structure of the brain and based on spiking artificial neurons and synapses.

Remarkable progress in neuromorphic computation has been made based on complementary metal-oxidesemiconductors (CMOS), both on the level of single devices and hardware architectures. However, in terms of scalability and energy efficiency, CMOS technologies will never be suitable for neuromorphic computation. To emulate nature, nanotechnology and materials science must offer new concepts - clear goals of the National Nanotechnology
Initiative (M. A. Meador, Nature Nanotech. 11, 401-402; 2016). In this direction, the recent discovery of memristive behaviour (J. Joshua Yang et al., Nature Nanotech. 8, 13-24; 2013) - a resistance state that depends on the previous history of applied voltages and currents, which is naturally suitable for implementing learning processes at the single-device level - has stimulated intensive basic research on possible materials and nanoscale devices possessing this property.

In this issue, on page 693, Tomas Tuma and colleagues now report a single nanoscale memristive device based on phase-change materials that mimics both the integrateand-fire functionality of the neuronal membrane and its intrinsically stochastic dynamics on the nanosecond timescale, and that has a remarkably low energy consumption. In spite of the limitations, the prospect of scalability of these devices means that we are a step closer towards a deep paradigm shift in computation.

\section{Hopes and anxieties}

\section{The uncertainty created by the result of the Brexit referendum will be damaging for science in the UK.}

On 23 June 2016, 52\% of people in the UK voted in favour of leaving the European Union (EU). Several weeks later and with a new government in place, there is still no certainty on what the separation will involve, and it may take years before it takes place.

As we highlighted in our July 2015 editorial (Nature Nanotech. 10, 565; 2015), science in the UK has historically benefited from EU membership, both in terms of funding and the large number of skilled European scientists working in the UK thanks to freedom of movement. The situation will not change immediately. Andrea Ferrari, professor at the University of Cambridge and chair of the EU Graphene Flagship management board, assured Nature Nanotechnology that the Graphene Flagship will continue to operate with a business-as-usual attitude at least until the official separation. For example, there is no reason why new members based at UK institutions should not be able to join the programme in the next few months. There is also no reason to believe that EU funding will decrease dramatically in the near future before a formal exit, whenever that will be.

But the uncertainty of what will happen after the separation is problematic. Although there is currently freedom of movement, it can be expected that fewer European scientists will want to move to the UK. It is also likely that technology and innovation companies will be cautious about investing in the UK until the situation is clarified. Even more worryingly, UK-based scientists will have to wait for the outcome of long negotiations to find out whether their participation in EU-wide projects will be, at best, similar to what it is now.

We remain convinced that science is one of the areas that will suffer as a result of the UK leaving the EU, and it is disheartening because scientific collaboration embodies fully the principle of peaceful cooperation to create a politically, economically and socially stronger continent, a principle that is at the very foundation of the EU. Perhaps the EU should engage more with its citizens to show them that it is still founded on this principle, rather than just being the technocratic and authoritarian institution that the growing number of Eurosceptics perceive it to be. 